Case report

\title{
Complex treatment of locally advanced squamous cell carcinoma of the parotid gland and secondary primary melanoma
}

\author{
Alina Chelmuș ${ }^{* 1,2}$, Liviu Dumitru Damian¹, Dragoș Pieptu ${ }^{1,2}$, Codrin Nicolae \\ Dobreanu $^{1}$
}

${ }^{1}$ Department of Plastic and Reconstructive Surgery, Regional Institute of Oncology, lași, Romania;

${ }^{2}$ "Grigore T. Popa” University of Medicine and Pharmacy, Iași, Romania

\begin{abstract}
Primary squamous cell carcinoma of the parotid gland accounts for $0.35 \%$ up to $9.8 \%$ of all parotid malignancies. In advanced stages, the gold standard is wide excision, lymphadenectomy and postoperative radiotherapy. Furthermore, head and neck cancers have an increased risk of secondary primary tumors development. Case report: A 82-year-old male underwent subtotal parotidectomy with facial nerve preservation for right parotid squamous cell carcinoma, in another department. Due to incomplete resection, 4 months later he presented with locoregional recurrence and cutaneous invasion. The patient underwent total parotidectomy en bloc with facial nerve, part of the external auditory meatus, posterior belly of digastric and part of sternocleidomastoid muscle with modified radical neck dissection, levels I-V. The defect was closed with ipsilateral pectoralis major myocutaneous flap, followed by a temporary lateral tarsorrhaphy after two months. The patient underwent adjuvant radiotherapy. One year after surgery, functional and aesthetic impairment of the facial nerve palsy were corrected: the lower face deficit by an orthodromic temporalis tendon transfer; lagophthalmos by lid loading with gold-weight. Postoperative outcome was satisfactory for the patient, with 3 years of postoperative evolution with no recurrence or metastasis. Concomitantly, the patient developed pT1a secondary primary melanoma on the skin island of the flap, which was excised. Conclusion: Patients with locoregional advanced parotid squamous cell carcinoma can benefit from wide excision with neck dissection and postoperative radiotherapy. When the facial nerve cannot be preserved, facial asymmetry can be improved through reanimation procedures. Secondary primary malignancies require proper treatment concomitantly with index tumor follow-up.
\end{abstract}

Keywords: parotid gland; squamous cell carcinoma; melanoma secondary tumor; facial reanimation; pectoralis major myocutaneous flap

\section{Introduction}

Salivary gland carcinoma is rare entity, accounting for up to $6 \%$ of head and neck cancers, with the parotid gland being the most frequent site among the major salivary glands $[1,2]$. In 2005, the World Health Organization

Received: July 2018; Accepted after review: August 2018; Published: September 2018.

*Corresponding author: Alina Chelmuș, Department of Plastic and Reconstructive Surgery, Regional Institute of Oncology, Nr 2-4 Henri Mathias Berthelot Street, 700483, Iași, Romania.

Email: ch alina 3@yahoo.com listed 24 different subtypes of salivary gland carcinomas [1, 3]. Primary squamous cell carcinoma (SCC) of the parotid gland is an uncommon tumor with aggressive behavior. The reported frequency of SCC ranges from $0.35 \%$ up to $9.8 \%$ of all parotid malignancies. [4]. Although for low-risk tumors a single modality treatment could achieve locoregional disease control, high-risk tumors have an aggressive behavior and require a multidisciplinary approach $[2,5]$. In advanced stages, the treatment of choice is surgical wide excision with neck dissection and 
postoperative radiotherapy in order to achieve better locoregional disease control [4, 5]. Although efforts should be made to preserve the facial nerve, when there is direct tumor invasion the nerve should be resected in order to leave no gross residual disease [5].

Secondary primary malignancies (SPM) are a particular feature of the head and neck cancers. Patients with head and neck squamous cell carcinoma have a high risk to develop SPM, most commonly in the same anatomic region [6, 7].

Herein, we report a $T_{4}$ parotid recurrent SCC that required wide excision en bloc with the facial nerve, neck dissection, primary reconstruction with a pedicle pectoralis myocutaneous flap and postoperative radiotherapy. The patient required multiple procedures to correct functional and aesthetic impairment caused by facial nerve palsy. Furthermore, one year after en bloc excision, the patient developed a SPM: $\mathrm{pT}_{1 \mathrm{a}}$ melanoma on the pectoralis flaps' skin island. To the authors' knowledge, this type of primary tumor association, parotid squamous cell carcinoma and secondary primary skin melanoma is reported for the first time.

\section{Case report}

An 82 year-old male diagnosed with right parotid squamous cell carcinoma in 2014, underwent subtotal parotidectomy with facial nerve preservation and sternocleidomastoid myoplasty in a different healthcare facility. Four months later, the patient was admitted in our clinic with local cutaneous invasion (Figure 1). CT-scan revealed extension to the lower wall of the external auditory meatus and the upper extremity of sternocleidomastoid muscle. The patient showed no significant medical history.
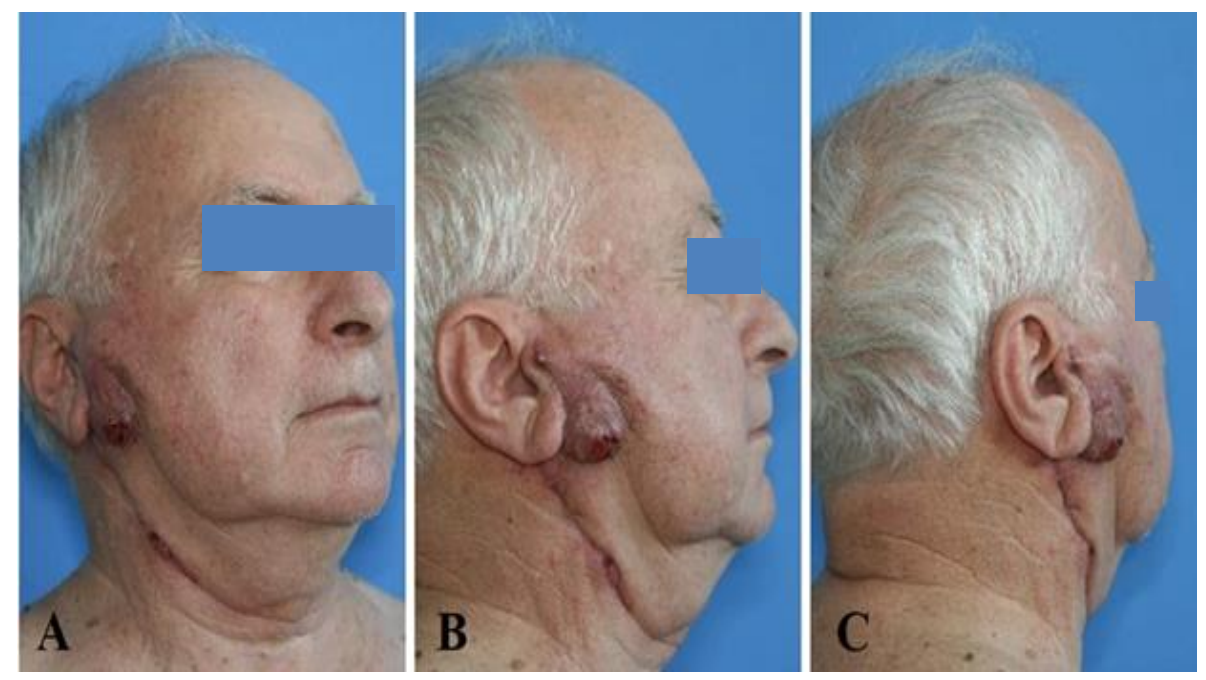

Fig. 1. Preauricular cutaneous tumor invasion: A. Right oblique anterior view; B. Right lateral view; C. Right oblique posterior view

The patient agreed to the informed consent for total parotidectomy associated with modified radical neck dissection. Under general anesthesia, the skin was incised circumferentially at $1 \mathrm{~cm}$ from tumor macroscopic borders, including the anterior region of the helix, tragus, partially the lobule of ear and the anteroinferior part of the external auditory meatus. The deep lobe of the parotid was excised en bloc with the facial nerve, due to tumor invasion. The excision extended to the masseter and medial pterygoid muscles and included the posterior belly of the digastric and the third upper part of the sternocleidomastoid muscle. The oncologic resection was associated with modified radical neck dissection, levels I-V with preservation of the spinal accessory nerve and internal jugular vein (Figure 2). The $6 / 9 \mathrm{~cm}$ defect was closed using an ipsilateral pectoralis major myocutaneous flap. The flap was passed into the defect through a 
subcutaneous tunnel created superficial to the clavicle (Figure 4A-B). The donor site was primarily closed. There were no major

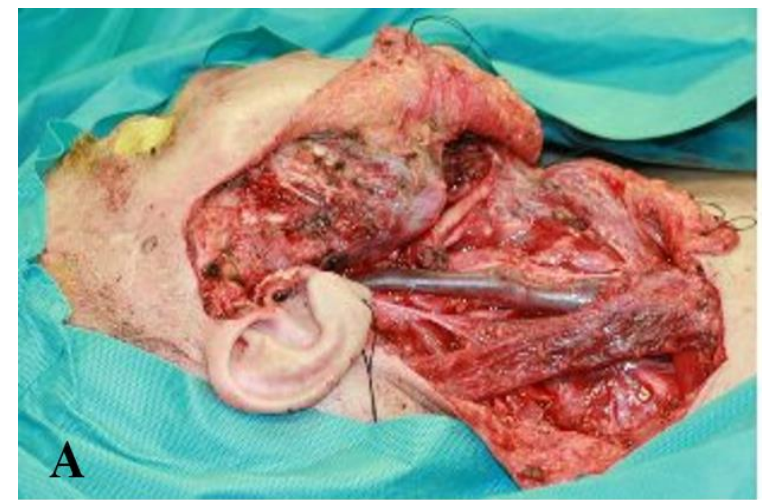

complications throughout the postoperative care and the patient was discharged after 11 days.

Fig. 2. A. The defect resulted after wide excision; B. En bloc tumor resection with $1 \mathrm{~cm}$ safety margins and neck dissection specimen

Two months later, the patient underwent a right temporary lateral tarsorrhaphy, to protect the cornea and reduce symptoms of exposure due to right facial nerve palsy. The patient received adjuvant radiotherapy, through IAMT technique. The patient was scheduled to receive 66 Gy in 33 fractions over $6 \frac{1}{2}$ weeks for the tumor site and $50 \mathrm{~Gy}$ in 25 fractions over 5 weeks for the right lateral cervical area. Due to grade IV radiation mucositis, swallowing difficulty and a $10 \%$ weight loss,

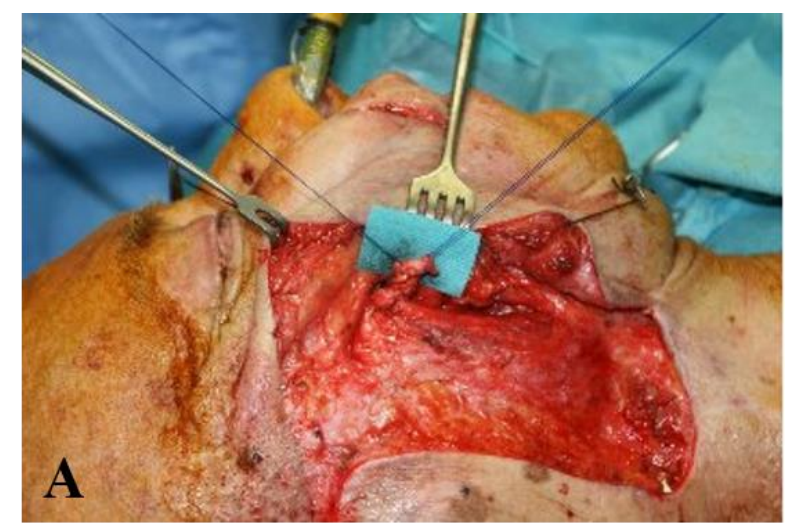

treatment was stopped at 62 Gy in 31 fractions over 6 weeks.

One year after surgery, procedures to correct functional and aesthetic impairment caused by facial nerve palsy were initiated. In first stage the patient was admitted to our clinic for an orthodromic temporalis tendon transfer. Under general anesthesia, the insertion tendon of temporalis muscle en bloc with part of the coronoid process was transferred through a subcutaneous tunnel and anchored to the right modiolus (Figure 3).

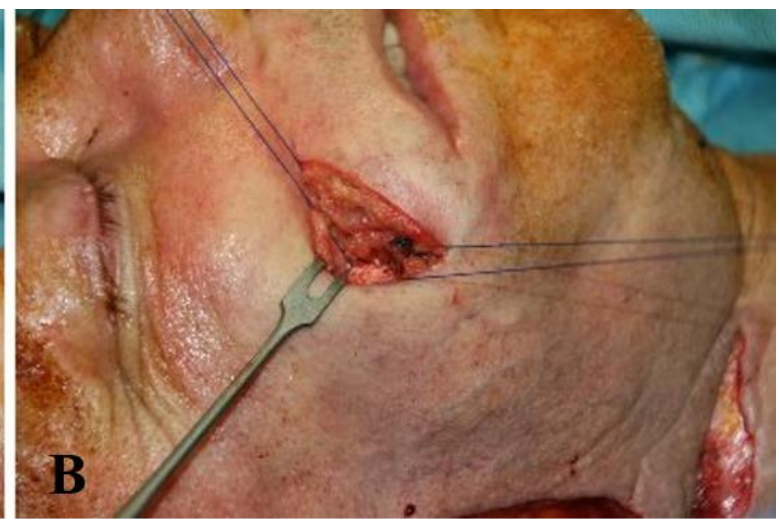

Fig. 3. Orthodromic temporalis tendon transfer: A. Temporalis tendon transfer; $B$. Tendon anchored to the right modiolus

At the same time, a $3 / 3 \mathrm{~mm}$ pigmented lesion was excised from the medial border of the pectoralis major myocutaneous flap. The final histology exam revealed a $\mathrm{pT}_{1 \mathrm{a}}$ melanoma, with Breslow's depth of $0.5 \mathrm{~mm}$ and Clark level III, for which follow-up according with the existing guidelines was performed (Figure 4A). Postoperative outcome was satisfactory, although no quantitative evaluation or questionnaires were applied (Figure 4C). 

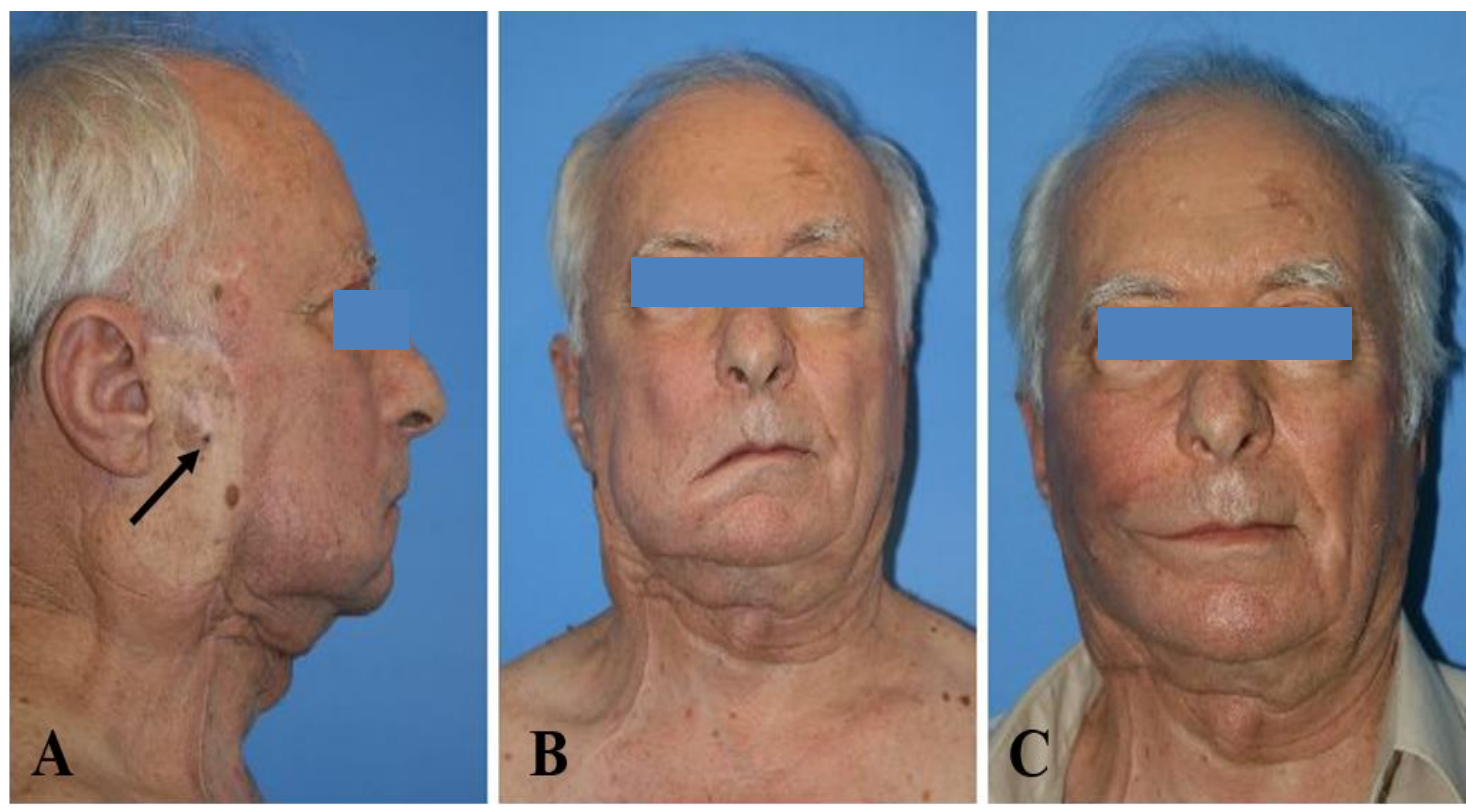

Fig. 4. A. Before orthodromic temporalis tendon transfer, with a pigmented lesion near the medial border of the pectoralis major myocutaneous flap, which the final histology examination revealed a $\mathrm{pT}_{1 \mathrm{a}}$ melanoma - right oblique anterior view; B. Before orthodromic temporalis tendon transfer, with drooping of the mouth corner on the right side - anterior view; C. Orthodromic temporalis tendon transfer outcome

After another 8 months and over 2 years after tumor resection with no locoregional recurrence or distant metastasis, the patient was admitted for lagophthalmos correction.
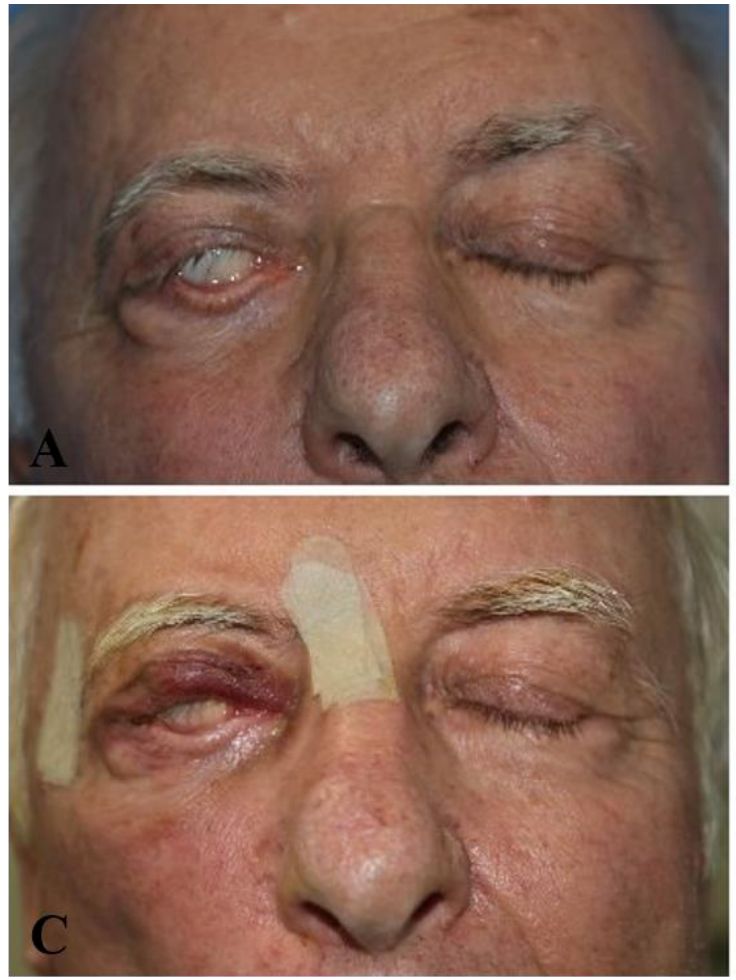

Under local anesthesia, the lateral tarsorrhaphy was reversed and a $1.2 \mathrm{~g}$ custom-made gold-weight was inserted through subtarsal approach (Figure 5).
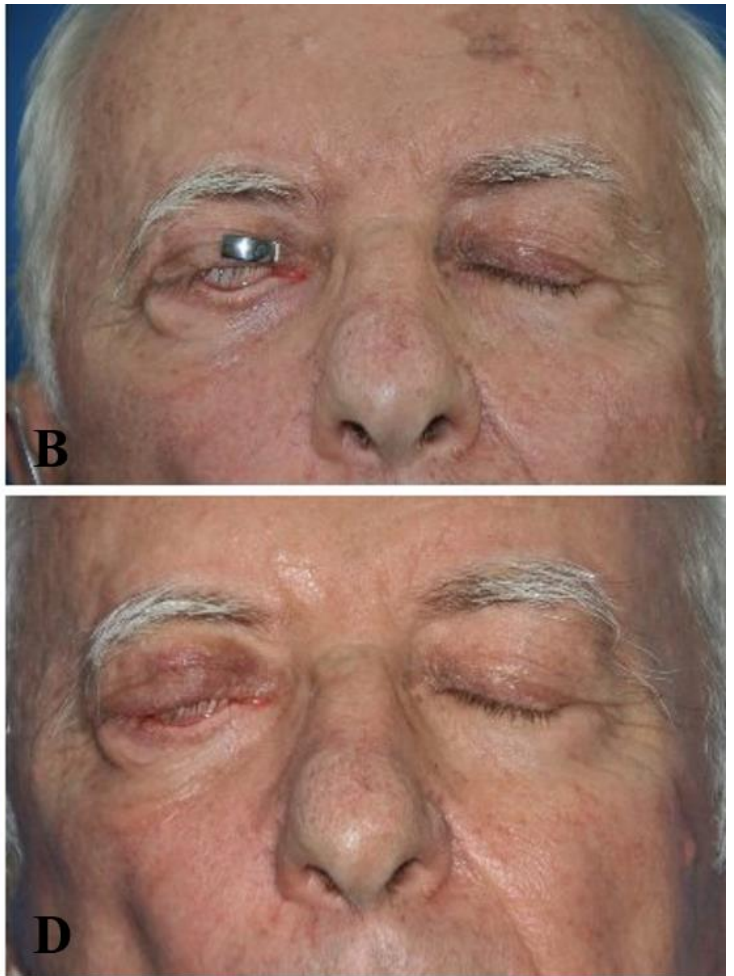

Fig. 5. A. Preoperative lid closure deficit; B. Gold eyelid weights measurement; C. One day after a $1.2 \mathrm{~g}$ custom-made gold-weight was inserted; D. 7 days after lid loading surgery with significant improvement of the lid closure deficit. 


\section{Discussions}

The management of head and neck cancer patients frequently implies a complex process, which requires a multidisciplinary approach, especially in advanced stages. Squamous cell carcinoma arising de novo in the parotid gland is rare, with an aggressive phenotype and a poor prognosis. Usually, the parotid gland is the site of metastasis from skin SCC [5, 8, 9]. In this case, the patient previously diagnosed with primary parotid squamous cell carcinoma, was admitted to our clinic 4 months after subtotal parotidectomy with facial nerve preservation due to local evolution with cutaneous invasion.

The National Comprehensive Cancer Network (NCCN) guideline for salivary gland tumor recommends surgery with adjuvant therapy in the case of incompletely resected tumors without prior radiotherapy, if the tumor is resectable [5].

The tumor was excised en bloc with part of the external ear, part of the sternocleidomastoid muscle and the posterior belly of the digastric. Due to macroscopic direct tumor invasion, the facial nerve was resected in order to leave no gross residual tumor and to achieve clear margins. Although clinical and CT-scan assessments did not reveal any enlarged lymph nodes, the patient underwent ipsilateral modified radical neck dissection, levels I-V.

While elective treatment for $\mathrm{N}_{0}$ stages disease remains controversial, the decision was based on the fact that the CT-scan revealed tumor extension to the upper extremity of the sternocleidomastoid muscle. The NCCN guideline considers in-continuity neck dissection necessary when there is direct extension of the primary tumor into the neck [5]. Furthermore, for clinical $\mathrm{N}_{0}$, there are studies that recommend neck dissection for high-grade and/or advanced tumors, such as $\mathrm{T}_{4}$. Moreover, the incidence of occult lymph node metastasis from salivary gland carcinomas can range from $10 \%$ up to $20 \%$ and advance tumor stage is correlated with occult disease [1]. Armstrong et al. reported a $24 \%$ risk of occult neck involvement among patients with neck $\mathrm{N}_{0}$ and $\mathrm{T}_{4}$ lesion [10].
The $6 / 9 \mathrm{~cm}$ defect required a good coverage due to the fact that the patient would undergo adjuvant radiotherapy. Considering the reconstructive ladder, the defect, postoperative radiotherapy and patient factors, direct closure, skin graft and local flaps were not an option for this case. Regarding free flap, which became the gold standard in head and neck reconstruction, this was a limited option in this case, especially due to patient related factors and the radiotherapy requirement [11]. Furthermore, by avoiding a microsurgical anastomosis, the operating time drops considerable [12, 13]. Our preferred coverage option in this case was an ipsilateral pectoralis major myocutaneous flap. The advantages of this flap include straightforward, adequate soft tissue volume, large skin paddle, versatility, reliability and robust vascular supply [12, 14]. Moreover, it is located outside the radiation field of the head and neck region [11]. Free flaps are the backup solutions for possible recurrence in demand for larger excision and more complex reconstruction.

When a locoregional recurrence without prior radiotherapy is completely resected, but has adverse features such as deep closure margins with macroscopic direct tumor invasion of the facial nerve, postoperative radiotherapy is indicated [5]. Furthermore, some studies recommend adjuvant therapy when there is deep lobe involvement and skin invasion, as in the case presented [15-18]. Postoperative radiotherapy improves the overall local control rate from $59 \%$ up to $82 \%$ $[15,19]$. The patient presented adverse features and was therefore graded as highrisk, requiring a dose of 60-66 Gy in 6-7 weeks [5]. Due to grade IV radiation mucositis, the treatment was stopped at 62 Gy over 6 weeks. Although the treatment was stopped, the patient received an appropriate dose according to guideline recommendations. Radiation-induced mucositis is one of the major side-effects of radiation therapy and a dose-limiting toxicity in head and neck cancer patients. Such a complication occurs in up to $80 \%$ of irradiated patients, out of which $56 \%$ are grade 3 and 4 . In $35 \%$ of the cases, reduction or cessation of irradiation treatment 
is required, as in the case hereby reported [2022].

Although during parotid cancer surgery efforts should be made to preserve the facial nerve, in the present case the facial nerve was resected due to macroscopic direct tumor invasion. Lagophthalmos represents the main ophthalmic sequela, which leads to chronic corneal exposure that increases the risk of ulcerations, with potential visual impairment $[23,24]$. Two months after the large excision, the patient underwent a temporary lateral tarsorrhaphy to protect the cornea and reduce the symptoms of exposure. At two years the lateral tarsorrhaphy was reversed and a $1.2 \mathrm{~g}$ custom-made gold-weight was used for lid loading. For lower face reanimation, the patient underwent an orthodromic temporalis tendon transfer at one year after en bloc excision, adapted to patient's age and etiology [25]. Orthodromic temporalis tendon transfer can be performed in patients with facial palsy secondary to cancer resection with low revision and complication rates. Furthermore, this procedure offers the possibility of dynamic movement. Although some studies report higher wound complication rate for this procedure in irradiated patients, our patient's postoperative course was uneventful [26, 27].

One year after en bloc excision the patient developed a $\mathrm{pT}_{1}$ a melanoma on the skin island of the pectoralis major myocutaneous flap, which was subsequently excised. The possibility of developing a second primary tumor is a well-known concept, particularly in head and neck cancer patients. It is not uncommon to develop a SPM, which in $80 \%$ of the cases is a squamous cell carcinoma $[6,7$, 28]. However, our patient had a squamous cell carcinoma as the index tumor, developed in the parotid gland, which is already a rare finding, and moreover, the SPM was a melanoma developed on the skin of the flap,

\section{References}

1. Wang $X$, Luo $Y$, Li M, Yan $H$, Sun M, Fan $T$. Management of salivary gland carcinomas - a review. Oncotarget 2017; 8(3):3946-3956.

2. Mifsud MJ, Burton JN, Trotti AM, Padhya TA. Multidisciplinary management of salivary gland cancers. Cancer Control 2016; 23(3):242-248. which is highly unusual. To our knowledge, such an association has never been reported so far. Furthermore, even if the melanoma developed on a prior irradiated site, it does not meet the criteria for a radio-induced malignancy [29]. Some studies suggest that radiotherapy of the index tumor site does not increase the risk of SPM development, although in this case the SPM developed in the irradiated area [6]. The treatment and follow-up were done according to the guidelines concomitantly with the index tumor follow-up.

\section{Conclusions}

Patients with locoregional recurrent parotid SCC benefit from wide excision associated with neck dissection and postoperative radiotherapy. Although facial nerve preservation is recommended, when there is direct tumor invasion, the nerve should be excised to yield clear margins. The facial asymmetry due to the facial nerve palsy can be improved through a combination of static and dynamic reanimation procedures. Even if radiotherapy can increase the risk of wound complications, it still remains at a low risk. Head and neck cancer patients have the risk of SPM development, which require proper management. Further follow-up and life-quality assessment is needed to establish long-term patient satisfaction.

\section{Consent}

Written informed consent was obtained from the patient for publication of this case report.

\section{Competing interests}

The authors have no conflict of interests to declare.
3. Thompson L. World Health Organization classification of tumours: pathology and genetics of head and neck tumours. Ear Nose Throat J 2006; 85:74. 
4. Lee S, Kim GE, Park CS, et al. Primary squamous cell carcinoma of the parotid gland. Am J Otolaryngol 2001; 22(6):400-406.

5. National Comprehensive Cancer Network. Head and Neck Cancers. NCCN Clinical Practice Guidelines in Oncology. Version 2. 2017.

[https://www.nccn.org/professionals/physician_ gls/default.aspx available at 10/17/2018]

6. Jones AS, Morar P, Phillips DE, Field JK, Husband D, Helliwell TR. Second primary tumors in patients with head and neck squamous cell carcinoma. Cancer 1995; 75(6):1343-1353.

7. Morris LG, Sikora AG, Patel SG, Hayes RB, Ganly I. Second primary cancers after an index head and neck cancer: subsite-specific trends in the era of human papillomavirus-associated oropharyngeal cancer. J Clin Oncol 2011; 29(6):739-746.

8. Flynn MB, Maguire $\mathrm{S}$, Martinez $\mathrm{S}$, Tesmer $\mathrm{T}$. Primary squamous cell carcinoma of the parotid gland: the importance of correct histological diagnosis. Ann Surg Oncol 1999; 6(8):768-770.

9. Giriyan S, Paragannavar V. Primary squamous cell carcinoma of parotid gland: common tumor at an uncommon site - a case report. Int $J$ Sci Res 2015; 4(10):520-522

10. Armstrong JG, Harrison LB, Thaler HT, et al. The indications for elective treatment of the neck in cancer of the major salivary glands. Cancer 1992; 69: 615-619.

11. Wei WI, Wei CY. Pectoralis Major Flap. In: Wei FC, Mardini S, editors. Flaps and reconstructive surgery. London: Elsevier, 2017; 396-415.

12. Liu $M$, Liu W, Yang $X$, Guo $H$, Peng $H$. Pectoralis major myocutaneous flap for head and neck defects in the era of free flaps: harvesting technique and indications. Sci Rep 2017; 7:46256.

13. Tripathi M, Parshad S, Karwasra RK, Singh V. Pectoralis major myocutaneous flap in head and neck reconstruction: An experience in 100 consecutive cases. Natl J Maxillofac Surg 2015; 6(1):37-41.

14. Patel K, Lyu DJH, Kademani D. Pectoralis major myocutaneous flap. Oral Maxillofacial Surg Clin N Am 2014; 26:421-426.

15. Sayan M, Vempati $P$, Miles $B$, et al. Adjuvant therapy for salivary gland carcinomas. Anticancer Res 2016; 36(8):4165-4170.
16. Nagliati M, Bolner A, Vanoni V, et al. Surgery and radiotherapy in the treatment of malignant parotid tumors: a retrospective multicenter study. Tumori 2009; 95(4):442-448.

17. Bell RB, Dierks EJ, Homer L, Potter BE. Management and outcome of patients with malignant salivary gland tumors. $J$ Oral Maxillofac Surg 2005; 63(7):917-928.

18. Cederblad L, Johansson S, Enblad G, Engstrom M, Blomquist $E$. Cancer of the parotid gland; long-term follow-up. A single centre experience on recurrence and survival. Acta Oncol 2009; 48(4):549-555.

19. Terhaard $\mathrm{CH}$, Lubsen $\mathrm{H}$, Rasch $\mathrm{CR}$, et al. The role of radiotherapy in the treatment of malignant salivary gland tumors. Int $J$ Radiat Oncol Biol Phys 2005; 61(1):103-111.

20. Maria OM, Eliopoulos N, Muanza T. Radiationinduced oral mucositis. Front Oncol 2017; 7:89.

21. Muanza TM, Cotrim AP, McAuliffe M, Sowers $A L$, Baum BJ, Cook JA et al. Evaluation of radiation-induced oral mucositis by optical coherence tomography. Clin Cancer Res 2005; 11(14):5121-5127.

22. Sonis ST, Elting LS, Keefe D, et al. Perspectives on cancer therapy-induced mucosal injury. Cancer 2004; 100(Suppl. 9): 1995-2025.

23. Vásquez LM, Medel R. Lagophthalmos after facial palsy: current therapeutic options. Ophthalmic Res 2014; 52:165-169.

24. Pereira MVC, Glória ALF. Lagophthalmos. Semin Ophthalmol 2010; 25(3):72-78.

25. Leedy JE. Facial reanimation. In: Janis JE, editor. Essentials of Plastic Surgery. St. Louis, Missouri: Quality Medical Publishing, Inc., 2007; 347-361.

26. Kejner AE, Rosenthal EL. Lower facial reanimation techniques following cancer resection and free flap reconstruction. Laryngoscope 2016; 126(9):1990-1994.

27. Griffin GR, Abuzeid W, Vainshtein J, Kim JC. Outcomes following temporalis tendon transfer in irradiated patients. Arch Facial Plast Surg 2012; 14:395-402.

28. Liao LJ, Chou HW, Wang CT, Chung CS, Lai MS. The impact of second primary malignancies on head and neck cancer survivors: a nationwide cohort study. PLoS One 2013; 8(4):e62116.

29. Singh GK, Yadav V, Singh $P$, Bhowmik KT. Radiation-induced malignancies making radiotherapy a "two-edged sword": a review of literature. World J Oncol 2017; 8(1):1-6. 